\title{
Translational and Implementation Research to Bridge Evidence and Implementation
}

\author{
Klaus Kraemer ${ }^{a}$ b Kesso Gabrielle van Zutphen ${ }^{a, c}$ \\ ${ }^{a}$ Sight and Life Foundation, Basel, Switzerland; ${ }^{b}$ Johns Hopkins University, Department of International Health, \\ Baltimore, MD, USA; ' Wageningen University \& Research, Department of Human Nutrition \& Health, Wageningen, \\ The Netherlands
}

\author{
Keywords \\ Implementation · Translational · Research · Science · Policy · \\ Programs · Malnutrition
}

\begin{abstract}
Background: The role of science in guiding interventions and programs and contributing to progress in achieving global targets is undeniable. In public health nutrition, biological research in the past century focused largely on single nutrients and provided the basis for addressing nutritional deficiencies. This focus has now expanded to consider evidence including, but not limited, to knowledge about food, diet, behavior, context, and culture. The complex double burden of malnutrition will need to be addressed through a wider lens that appreciates the multiple and interrelated facets that underpin it. Summary: Despite the acknowledged importance of translational research in improving nutritional outcomes, significant gaps remain in the process leading from science to practice. This article sheds light on 2 examples that demonstrate this, namely, anemia and stunting. Further, much work is still required to translate the current evidence base into effective actions that result in impact at scale, pointing toward the need for more implementation research in nutrition. Key Messages: While discoveries may take time to surface and implementers are impatient to address the challenge at hand, it is essential to identify and de-
\end{abstract}

\section{KARGER}

E-Mail karger@karger.com www.karger.com/anm

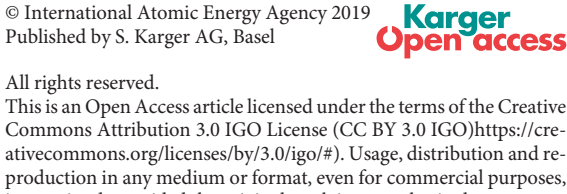
Commons Attribution 3.0 IGO License (CC BY 3.0 IGO)https://creativecommons.org/licenses/by/3.0/igo/\#). Usage, distribution and reproduction in any medium or format, even for commercial purposes, is permitted, provided the original work is properly cited. ploy the best available evidence while continuously advancing the evidence base, and to seek the right balance between action and inaction.

(C) International Atomic Energy Agency 2019 Published by S. Karger AG, Basel

\section{Translating Science into Practice}

Translational research can be defined as the "process of applying knowledge from basic biology and clinical trials to techniques and tools that address critical medical needs" [1]. This type of research specifically enhances the adoption of evidence-based practices in real-world settings to reach broad populations. The conversion of conclusive research into practice must be at the core of the nutrition community's quest to improve nutrition outcomes. In public health nutrition, biological research in the past century largely focused on single nutrients and provided the basis for addressing nutritional deficiencies. However, this focus has now expanded to consider a broader body of evidence. In fact, we increasingly understand what constitutes nutrition

The article is part of the Proceedings of the International Symposium on Understanding the Double Burden of Malnutrition for Effective Interventions organized by the International Atomic Energy Agency (IAEA) in cooperation with United Nations Children's Fund (UNICEF) and World Health Organization (WHO) (10-13 December 2018, Vienna, Austria).
Dr. Klaus Kraemer

Sight and Life Foundation PO Box 2116

$\mathrm{CH}-4002$ Basel (Switzerland)

E-Mail klaus.kraemer@sightandlife.org 
and health using knowledge about food, diet, behavior, and socioeconomics, and we are even starting to appreciate the biology of emotional health, stress, and anxiety in pregnancy as a predictor of child development and long-term health outcomes. Addressing the emerging multiple burden of malnutrition (undernutrition, overweight/obesity, and micronutrient deficiencies) requires such an approach as it depends on in-depth understanding of context and of complex biology to inform comprehensive strategies that integrate nutrition across sectors (e.g., health, agriculture, WASH, education, social protection).

Translational research also refers to the transfer of knowledge to the development of evidence-based guidelines [2]. More precisely, it involves how knowledge of biology can be translated into effective interventions to address malnutrition in all its forms, and how scientists can align their research with policy makers' priorities to ensure that biological evidence is translated into coherent policies. One way of doing so is through the issue of technical guidelines or systematic reviews by relevant institutions and/or expert groups, which serve as a facilitator or catalyst for evidence uptake at policy and program level. Additionally, international and national events can serve as platforms for dialogue and knowledge-sharing between the scientific community, country policy makers, and implementers. Further steps to support the linkage between biology and programs should involve strategic communication between the different players; the translation of advanced biological evidence into effective interventions; the identification of "entry points" for stimulating dialogue and building trust; and proposed models of collaboration between scientists and implementers that demonstrate program progress and accelerate the attainment of specific, measurable, achievable, realistic, and time-bound goals.

Despite the abovementioned importance of biology in improving nutritional outcomes for populations, there remain significant gaps in the process leading from science to practice, one of which is apparent through the example of anemia.

\section{The Need to Understand the Biology: The Example of Anemia}

One example that reflects the nutrition community's reservations on using biological knowledge is anemia. As underlined in the 2018 Global Nutrition Report, no country is on track to meet the global target of a $50 \%$ reduction of anemia in women of reproductive age [3]. The problem appears intractable with the prevalence of anemia even in-

Bridging Evidence and Implementation creasing slightly from $31.6 \%$ in 2000 for girls and women aged $15-49$ to $32.8 \%$ in 2016 [3]. In pregnant women, the global prevalence has decreased only slightly, from $41.6 \%$ in 2000 to $40.1 \%$ in 2016 [3]. Within the nutrition community and beyond, there is a widely prevalent misconception that anemia is mainly related to iron deficiency. Moreover, the knowledge gained over the last decade about iron biology and hepcidin in particular has not been adequately deployed. Hepcidin is the master regulator of iron homeostasis and its expression is downregulated by low iron status and active erythropoiesis and upregulated by iron overload, inflammation and/or infection. This suggests that interventions aimed at reducing inflammation and infections are probably as important as dietary interventions to effectively address anemia [4]. According to recent estimates, only $25 \%$ of preschoolers suffer from anemia due to iron deficiency and only $37 \%$ of women's anemia is due to iron deficiency, which reflects the need to uncover and address additional causes [3]. The etiology of anemia involves genetic factors (e.g., thalassemia, sickle cell disease) and nutrient deficiencies other than iron, along with infection and inflammation. Moreover, it is essential to note that for people affected by a type of anemia other than iron deficiency anemia, iron supplements and fortificants are at best ineffective and, in the worst case, may be harmful (increasing oxidative stress, feeding parasites, negatively affecting the microbiome, etc.). The etiology of anemia must therefore be understood and taken into consideration when designing interventions aimed at reducing anemia.

There are also instances where the nutrition community has steered away from evidence by oversimplifying knowledge. The Nutrition ARISE (Adding Responsive Indicators to Stunting to Expand Early Life Nutrition and Development Achievement) expert consultation held in Boston, USA, in 2018 aspired to address some of these issues for childhood stunting [5].

\section{The Need to Embrace Complexity: The Example of Stunting}

Linear growth is strongly associated with a variety of different outcomes including, but not limited to, child development, schooling, and productivity [6]. Considering this relationship, the development community has come to focus primarily on the outcome (i.e., stunting) rather than interventions that lead to improvements in predisposing factors for stunting (e.g., sanitation, education, etc.). This has led to a narrow focus on reducing stunting in development. When firefighters come to extinguish a 


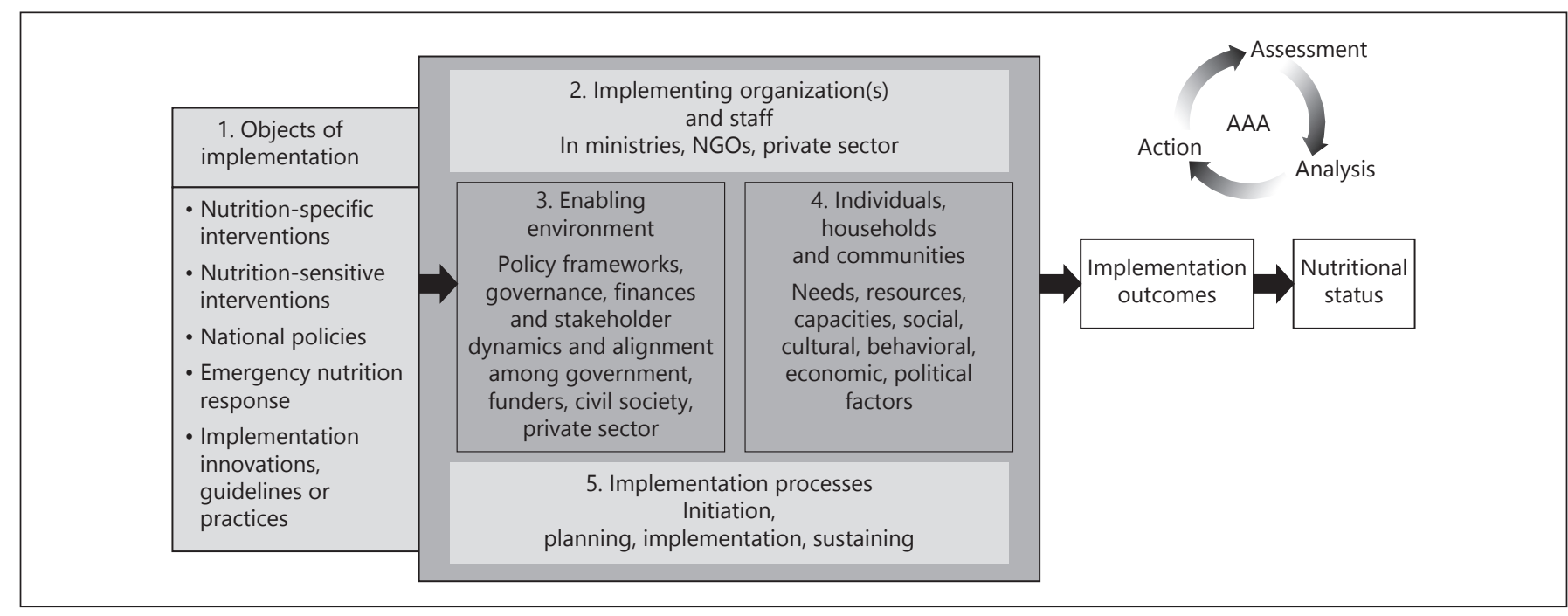

Fig. 1. The 5 domains whose characteristics, capacities, dynamics and fit affect implementation quality. Reproduced with permission from Tumilowicz et al. [10].

fire, they do not come to turn off the smoke detectors, but to extinguish the fire [7]. This analogy helps clarify the problem. In a sense, the predisposing factors that limit growth (i.e., the fire), such as poor micronutrient intake, repeated intestinal infections, unhealthy microbiome, and poor maternal education, may respond to nutrition interventions much faster and better than linear growth (i.e., the smoke detector) and actually have a broader impact on maternal and child nutrition, even if stunting is not the primary outcome [7]. However, interventions must be conducted with an understanding of the potential impact they may have on growth given the complex sequelae of factors promoting linear growth. Recently, a systematic analysis of 137 developing countries identified 18 key risk factors of stunting that included teenage pregnancy and short birth spacing, fetal growth restriction, preterm birth, as well as environmental factors (WASH, use of biomass fuel) being the most prominent [8]. Moreover, one important risk factor for stunting often not discussed in the nutrition community is maternal smoking [9]. Clearly, attention to stunting has brought unprecedented global support for nutrition and is an important accomplishment. Yet to maintain this momentum, the complexity of stunting needs to be acknowledged and appropriately taken into consideration when designing and rolling out policies and programs.

While the examples of anemia and stunting highlight how too much attention on the primary outcome can limit action on equally important and perhaps more pliable associated outcomes, a real shift in research and program evaluations is needed. Simply, much work is still required to translate the current evidence base into effective actions that result in impact at scale, which points toward the need for more implementation research in nutrition.

\section{Filling the Implementation Gap: The Role of Implementation Research}

The gap between what is known about efficacious interventions and what is actually achieved in real-world settings, also referred to as the "implementation gap," has spurred interest in developing and applying the science of implementation in nutrition [10]. Implementation research, a component of implementation science, is required to move from present levels to desired levels of program impact. It refers to "a variety of methods of assessment, inquiry, and formal research whose purpose is to systematically assess, build on strengths, and address potential weaknesses within and between the many factors that affect implementation or the phases of implementation" [10]. These many factors can be categorized into 5 domains, namely: the intervention, policy or innovation being implemented; the implementing organization(s); the enabling environment of policies and stakeholders; the individuals, households, and communities of interest; and the strategies and decision processes employed at various stages of the implementation process, as illustrated in Figure 1 [10]. Successfully aligning these domains through implementation research requires a culture of inquiry, 
learning, and response among program implementers, along with evaluation; continuity of funding for implementation research; an action-oriented mission among the research partners; and the resolution of tensions between research and program implementation [10]. The recently established Society for Implementation Science in Nutrition was formed with the aim of initiating these changes [10].

\section{Conclusion}

The role of science in guiding interventions and programs and contributing to progress in achieving global targets is undeniable. While discoveries may take time to surface and implementers are impatient to act to address the challenges at hand, it is essential not to lose momentum and to keep progressing in the fight against all forms of malnutrition. Identifying and deploying the best available evidence while continuously advancing the overall evidence base is therefore crucial. Of course, large-scale programs without adequate evidence for potential benefits should not be rolled out, but the right balance between action and inaction must be sought [11]. Adopting a systems-thinking approach whereby the interrelated cultural and socioeconomic system, the food system, the biological system, and others can join forces to analyze data may be helpful for piloting and testing varying scenarios [12]. Additionally, different skillsets (bioinformatics, engineering, modeling) can add value and accelerate the generation of evidence [11].

Bridging biological evidence to effective programs and implementation also requires a continuous two-way dialogue between scientists and implementers, so that the science can be implemented while remaining responsive to implementers' needs and challenges [13]. Many programs fail due to poor implementation and low coverage. It is therefore important to take advantage of evidence that is readily available, but has failed to be implemented yet and/ or failed to be adequately implemented to a point where impact can be achieved [7]. The tools to address implementation barriers are available to the research community, but commitment from programs to invest in this type of research is still lacking [14]. Finally, double duty interventions and programs ought to be integrated in a wider multisectoral framework [15]. However, to get sectors enthusiastic and engaged about nutrition is not an easy task. Specific, measurable, achievable, realistic, and time-bound outcomes that are attainable, feasible, and pragmatic need to be defined in a way that makes sense to the sectors in question. For example, poverty reduction for the social protection sector and access to healthy diets for the agricultural sector [7].

\section{Acknowledgments}

The authors would like to thank Daniel Hoffman, Cornelia Loechl, Theodora Mouratidou, and Pernille Kaestel for their valuable suggestion to improve the manuscript.

\section{Statement of Ethics}

The authors have no ethical conflicts to disclose for this review because there were no humans or animals involved directly.

\section{Disclosure Statement}

The authors have no conflicts of interest to declare in relationship to the content of the manuscript. K.K. is a founding member and former board member of the Society for Implementation Science in Nutrition.

\section{Funding Sources}

The authors did not receive any specific funding for the production of this paper, nor for the IAEA's International Symposium on Understanding the Double Burden of Malnutrition in 2018. During the symposium, K.K. presented at the panel session on "Bridging the evidence - how to strengthen the link between biology and implementation for sustainable action?" upon which this paper is based. Sight and Life Foundation financially contributed to the symposium. The contributions have been used to support the attendance to the symposium of participants with low financial resources and were also used to fund invited speakers and hospitality.

Open access provided with a grant from the International Atomic Energy Agency.

\section{Author Contributions}

K.K. conceptualized the vision and outline of the manuscript. K.G.vZ. led the drafting of the manuscript and integration of input. Both authors read and approved the draft.

\section{Disclaimer}

The statements, opinions and data contained in this publication are solely those of the individual authors and contributors, not of the publishers and the editor(s), and do not necessarily reflect the views of the cooperating organizations, IAEA, UNICEF and WHO. The use of particular designations of countries or territories does not imply any judgement by the cooperating organizations, as to the legal status of such countries or territories, of their authorities and institutions or of the delimitation of their boundaries. The mention of names of specific companies or products (whether or not indicated as registered) does not imply any intention to infringe proprietary rights, nor should it be construed as an endorsement or recommendation on the part of the cooperating organizations. 


\section{References}

1 Davis UC. What is Translational Research? [internet]. 2018 [cited February 7, 2019]. Available from: https://www.ucdavis.edu/ one-health/translational-research/.

2 Zoellner J, Van Horn L, Gleason PM, Boushey CJ. What is translational research? Concepts and applications in nutrition and dietetics. J Acad Nutr Diet. 2015 Jul;115(7):1057-71.

3 Development Initiatives. 2018 Global Nutrition Report: Shining a light to spur action on nutrition. Bristol, UK: Development Initiatives; 2018.

4 Prentice AM. Clinical implications of new insights into hepcidin-mediated regulation of iron absorption and metabolism. Ann Nutr Metab. 2017;71 Suppl 3:40-8.

5 Sight and Life. Nutrition ARISE - New Frontiers for Public Health Nutrition. Sight Life Magazine. 2018; (32)1.

6 Black RE, Victora CG, Walker SP, Bhutta ZA, Christian P, de Onis M, et al.; Maternal and Child Nutrition Study Group. Maternal and child undernutrition and overweight in low-income and middle-income countries. Lancet. 2013 Aug;382(9890):42751.

7 Leroy J. [Panel session on Bridging the evidence - how to strengthen the link between biology and implementation for sustainable action] IFPRI, Washington. 2018.

8 Danaei G, Andrews KG, Sudfeld CR, Fink G, McCoy DC, Peet E, et al. Risk Factors for Childhood Stunting in 137 Developing Countries: A Comparative Risk Assessment Analysis at Global, Regional, and Country Levels. PLoS Med. 2016 Nov;13(11):e1002164.

9 Quelhas D, Kompala C, Wittenbrink B, Han Z, Parker M, Shapiro M, et al. The association between active tobacco use during pregnancy and growth outcomes of children under five years of age: a systematic review and metaanalysis. BMC Public Health. 2018 Dec; 18(1): 1372.

10 Tumilowicz A, Ruel MT, Pelto G, Pelletier D, Monterrosa EC, Lapping K, et al.; Society for Implementation Science in Nutrition. Implementation Science in Nutrition: Concepts and Frameworks for an Emerging Field of Sci- ence and Practice. Curr Dev Nutr. 2018 Oct; 3(3):nzy080.

11 Udomkesmalee E. [Panel session on Bridging the evidence - how to strengthen the link between biology and implementation for sustainable action] Mahidol University, Bangkok. 2018.

12 Kraemer K. [Panel session on Bridging the evidence - how to strengthen the link between biology and implementation for sustainable action] Sight and Life, Basel. 2018.

13 Neufeld L. [Panel session on Bridging the evidence - how to strengthen the link between biology and implementation for sustainable action] GAIN, Geneva. 2018.

14 Pelto G. [Panel session on Bridging the evidence - how to strengthen the link between biology and implementation for sustainable action] 2018.

15 Chowdhury J. [Panel session on Bridging the evidence - how to strengthen the link between biology and implementation for sustainable action] Nutrition International, Ottawa. 2018. 